\title{
Otimização do Processo de Soldagem FCAW Usando o Erro Quadrático Médio Multivariado
}

\author{
(FCAW Welding Process Optimization Using the Multivariate Mean Square Error)
}

\author{
Emerson José de Paiva ${ }^{1}$, Lucilene de Oliveira Rodrigues ${ }^{1}$, Sebastião Carlos da Costa ${ }^{1}$, Anderson Paulo de Paiva ${ }^{1}$, Pedro Paulo \\ Balestrassi ${ }^{1}$ \\ ${ }^{1}$ Universidade Federal de Itajubá - UNIFEI, - Itajubá, MG, Brasil; emersonjpaiva@gmail.com
}

\begin{abstract}
Resumo
Encontrar um conjunto ótimo de parâmetros para um processo de soldagem é uma tarefa pouco trivial, face às múltiplas características exigíveis ou desejáveis que devem ser analisadas. Além disso, a negligência da estrutura de variância-covariância destas características na otimização pode conduzir a ótimos inadequados. Com o intuito de auxiliar na busca desses parâmetros, um método para otimização multiobjetiva, desenvolvido para o estudo do processo de soldagem FCAW (do inglês Flux Cored Arc Welding), utilizando-se arames tubulares, baseado no conceito de Erro Quadrático Médio Multivariado, será apresentado. Trata-se de uma abordagem combinada da Metodologia de Superfície de Resposta, Projeto de Experimentos e Análise de Componentes Principais, na tentativa de localizar valores próximos a alvos especificados, para cada uma das características estudadas (Penetração, Taxa de deposição, Rendimento, Índice de convexidade e Diluição), considerando-se as variáveis de processo expressas em função da tensão (V), velocidade de alimentação do arame (Va) e da distância do bico de contato-peça (d). Os resultados obtidos apontam para uma boa adequação desta proposta.
\end{abstract}

Palavras-chave: Erro Quadrático Médio Multivariado(EQMM). FCAW. Projeto e Análise de Experimentos. Metodologia de Superfície de Respostas.

\begin{abstract}
The optimization of welding processes is not a trivial task, mainly due to the great number of exigible and desirable characteristics that must be analyzed. Moreover, the optimization of a welding process with multiple characteristics without to consider the variance-covariance structure, may lead to inadequate optimum. To help in this task, a method of multiobjective optimization based in the Multivariate Mean Square Error applied in the study of multiple correlated characteristics of a FCAW (Flux Cored Arc Welding) welding process will be presented. This method characterized by a combined approach based in the Response Surface Methodology, Design of Experiments and Principal Components Analysis consisted in an attempt to achieve the nearest values to specific targets, for each studied characteristic (penetration, deposition rate, deposition efficiency, convexity index of the weld bead and dilution) considering the welding variables expressed in function of welding voltage $(V)$, wire feed speed (Va) and the contact tip to workpiece distance (d). The results point out a good adequacy of the proposed method.
\end{abstract}

Key-words: Multivariate Mean Square Error (MMSE); FCAW; Design of Experiments; Response Surface Methodology (RSM).

\section{Introdução}

Num processo de soldagem é desejável que se determinem parâmetros adequados para sua realização, com a máxima acurácia possível. A busca por esses parâmetros, entretanto, passa por um processo de otimização de múltiplas variáveis, representando múltiplas características de qualidade, exigíveis ou desejáveis, para um determinado processo ou produto, constituindo-se em uma tarefa de difícil execução e de eficácia improvável. Segundo Wu [1] e Khuri e Cornell [2], a presença de possíveis

(Recebido em 29/07/2009; Texto Final em 19/03/2010).

Publicado com permissão da ABCM - Associação Brasileira de Engenharia e Ciências Mecânicas - Artigo originalmente apresentado no $5^{\circ}$ COBEF, Belo Horizonte, MG, Abril de 2009 relacionamentos de dependência (ou correlação) entre essas várias características de saída dos processos, podem interferir na determinação desses parâmetros e conduzir o processo a ótimos inapropriados, resultando em conclusões equivocadas e sem sentido. Segundo Khuri e Conlon [3] e Bratchell [4] esta inadequação está associada à insuficiência do método dos mínimos quadrados ordinários em estimar os coeficientes das múltiplas respostas correlacionadas simultaneamente em função da forte influência das estruturas de correlação sobre as funções de transferência utilizadas.

Nesse sentido, o presente trabalho apresenta a aplicação de uma abordagem empregada por Paiva [7] a partir de uma extrapolação multivariada do método proposto por Köksoy e Yalcinoz [5], Lin e Tu [6] para utilização do Erro Quadrático Médio (EQM) na otimização simultânea de média e variância, com o objetivo de se determinar parâmetros ideais para os processos de manufatura. Com as adaptações propostas, o método 
denominado de EQMM (Erro Quadrático Médio Multivariado) é capaz de construir um modelo matemático com um conjunto adequado de parâmetros ótimos, gerado a partir das estruturas de correlação existentes entre as respostas, identificando essas estruturas antes que se construa tal modelo.

O método EQMM proposto por Paiva [7] apresenta um framework para a aplicação de diversas técnicas e metodologias tentando minimizar os esforços na busca de parâmetros adequados dos processos de manufatura.

Segundo Paiva [7], a otimização multivariada de tais parâmetros passa pela aplicação combinada de diferentes metodologias, como o Planejamento e Análise de Experimentos (DOE - Design of Experiments), a Metodologia de Superfície de Resposta (MSR) e a Análise de Componentes Principais (ACP) em problemas do tipo NTB (Nominal-the-best). Neste caso, utiliza-se o DOE para se estudar o comportamento das variáveis; a MSR para modelar as funções de aproximação de pontos de ótimo, geralmente encontrados em regiões dotadas de curvatura, de acordo com sua convexidade; e a ACP para a construção do índice EQMM, cujo objetivo é minimizar as distâncias entre as respostas e seus respectivos alvos e variâncias.

Com o objetivo de discutir a eficiência da aplicação desta metodologia e por considerar que tais características são típicas dos processos de soldagem, um caso de otimização multiobjetivo de múltiplas características correlacionadas ao processo de soldagem FCAW, será investigado. Vale ressaltar que os resultados obtidos não objetivam mostrar um avanço tecnológico do processo, mas sim a possibilidade do método ser aplicado em outros processos de manufatura similares. Tanto a metodologia proposta, quanto o caso investigado serão descritos nas próximas seções desse trabalho.

\section{2, Metodologias Combinadas}

Realizar a otimização de múltiplas características, considerando a estrutura de correlação, porventura existente, passa pela aplicação de metodologias combinadas que, segundo o modelo proposto por Paiva [7], devem ser empregadas para desenvolver os experimentos, encontrar o modelo quadrático representativo do problema, aglutinar as múltiplas funções objetivo e detectar a estrutura de correlação. Constatando a existência de correlação, deve-se proceder à análise de componentes principais, que aqui tem papel importante, pois revela estruturas de correlação, mesmo que latentes; encontrar os modelos quadráticos dos componentes principais; determinar os limites de especificação em termos de componentes principais, aplicando-se, finalmente, a formulação do Erro Quadrático Médio Multivariado.

A Figura 1 exibe o fluxo de procedimentos para aplicação das diversas metodologias estatísticas e matemáticas combinadas e obtenção dos parâmetros ideais pelo modelo EQMM.

A seguir, serão apresentadas as metodologias envolvidas para essa abordagem de otimização multivariada.

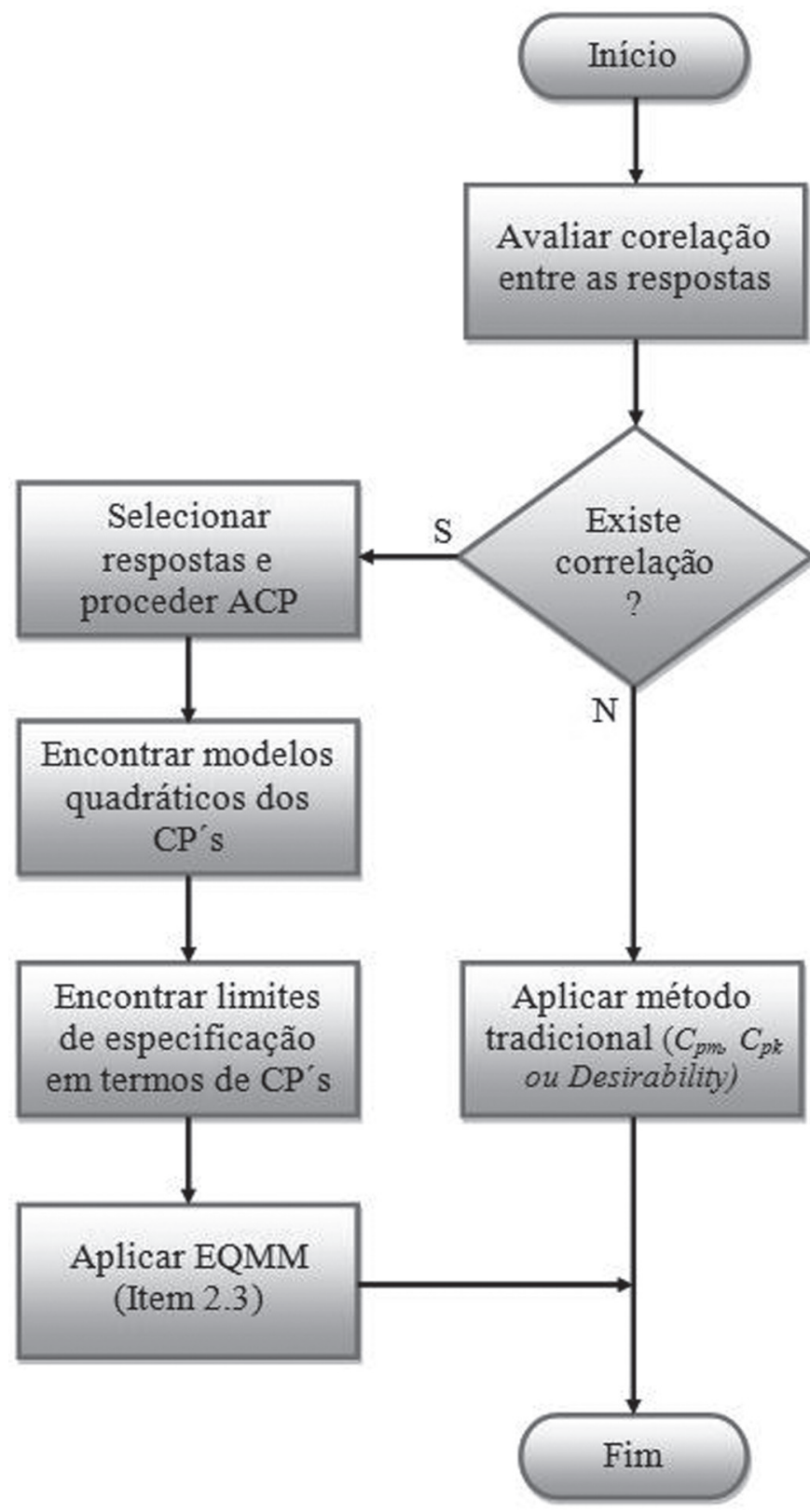

Figura 1. Fluxo de procedimentos para obtenção do EQMM.

\subsection{Planejamento e Análise de Experimentos}

A metodologia de Planejamento e Análise de Experimentos (DOE) é uma das principais estratégias disponíveis para a melhoria de processos. A partir de uma análise sistematizada acerca de um problema, busca-se avaliar a magnitude de várias fontes de variação que influenciam um processo. Segundo Montgomery [8], o processo deve se iniciar com a identificação e seleção dos fatores que possam contribuir para a variação, proceder-se, em seguida, à seleção de um modelo que inclua os fatores escolhidos, determinar seus níveis e planejar experimentos eficientes para estimar seus efeitos.

Conduzir adequadamente os experimentos, de acordo com o planejamento elaborado, garante o sucesso do problema em estudo ou, no mínimo, evita os riscos de insucesso da 
experimentação não planejada. Durante a sua condução, devese estar atento para detectar as anormalidades ocorridas, além de documentá-las para posterior análise, quando, então, serão estimados os fatores incluídos no modelo utilizando métodos estatísticos adequados, culminando na inferência, interpretação, discussão dos resultados, além da recomendação de melhorias, quando necessário.

Uma vez selecionados os fatores e seus respectivos níveis, gera-se uma combinação desses fatores sob a forma de arranjos experimentais. $\mathrm{O}$ arranjo mais comum é o fatorial completo, para o qual o número de experimentos é igual ao número de níveis experimentais, elevado ao número de fatores. No caso típico de fatoriais em dois níveis, o número de experimentos é dado por $N=2^{k}$. Fatoriais completos cobrem todo o espaço experimental. Entretanto, devido ao seu crescimento exponencial, arranjos com grande número de fatores podem tornar um processo de experimentação inviável. Para esses casos, Montgomery e Runger [9] afirmaram que, se houver pouco interesse nas interações, pode-se negligenciá-las, gerando-se frações do experimento completo sem comprometer, entretanto, a detecção da presença de fatores influentes.

O ponto de ótimo de um processo está, no entanto, geralmente associado a pontos estacionários, só podendo ser obtidos em superfícies ou funções não-lineares (em geral curvilíneas, côncavas, convexas ou mistas). Para tanto, uma evolução natural da prática experimental é migrar dos arranjos fatoriais para a Metodologia de Superfície de Resposta (MSR). A MSR é uma coleção de ferramentas matemáticas e estatísticas utilizada para modelar e analisar problemas, que são influenciadas por inúmeras variáveis e para as quais se desejam respostas, em geral, quadráticas [8]. Geralmente, o relacionamento entre as variáveis dependentes e independentes é desconhecido e o que se procura é encontrar uma razoável aproximação do relacionamento real entre as respostas (y) e o conjunto de variáveis independentes $(x)$. Como qualquer função, mesmo que desconhecida, pode ser aproximada por uma Série de Taylor [8], o truncamento desta série em seu termo quadrático gera a função não-linear denominada de "Superfície de Resposta". Portanto, se existir curvatura em um sistema, então a função de aproximação mais usada para um modelo de segunda ordem é a apresentada pela Equação 1:

$$
y=\beta_{0}+\sum_{i=1}^{k} \beta_{i} x_{i}+\sum_{i=1}^{k} \beta_{i} x_{i}^{2}+\sum_{i<j} \sum \beta_{j} x_{i} x_{j}+\varepsilon
$$

Onde: $\beta$ é o coeficiente polinomial, $k$ é o número de fatores e $\varepsilon$ é o erro aleatório. Os parâmetros $\beta$ do modelo podem ser estimados através do método dos Mínimos Quadrados Ordinários que, em forma matricial, podem ser representados pela Equação 2:

$$
\hat{\beta}=\left(X^{T} X\right)^{-1} X^{T} Y
$$

Na equação (2), $X$ é a matriz de fatores codificados e $Y$ é a resposta de interesse.
O principal arranjo experimental para o ajuste de "Superfícies de Resposta" é o CCD (Central Composite Design). Segundo Montgomery [8], parte deste arranjo é formado por um arranjo fatorial (completo ou fracionado), seguido da adição de pontos centrais e pontos axiais. Os pontos centrais equivalem aos pontos médios dos níveis dos fatores quantitativos e os axiais, distanciam-se dos centrais à razão constante de $\rho=\sqrt[4]{2^{k}}$. Este será o arranjo utilizado neste trabalho.

\subsection{Superfície de Resposta Dual}

Problemas multidimensionais do tipo "Nominal é melhor" (NTB - Nominal The Best) são aqueles em que se procura minimizar a distância entre várias respostas $\left(Y_{i}\right)$ e seus respectivos alvos $\left(\zeta_{Y}\right)$, concomitantemente com a redução de suas variâncias.

Para se alcançar esses objetivos, Vining e Myers [10] propuseram a Metodologia de Superfície de Resposta Dual, como forma de se atingir os alvos propostos para cada característica de qualidade envolvida, baseado numa superfície de resposta para a média $\left(\omega_{\mu}\right)$ e outra para a variância $\left(\omega_{\sigma^{2}}\right.$, ambas escritas como um

polinômio de segunda ordem. Lin e Tu [6] propuseram a combinação destas duas funções através da minimização do Erro Quadrático Médio (EQM), como critério de otimização simultânea de média e variância, conforme demonstra a Equação 3.

$$
E Q M=\left(\hat{\omega}_{\mu}-\zeta\right)^{2}+\hat{\omega}_{\sigma}^{2}
$$

Para múltiplas respostas, entretanto, duas estratégias podem ser adotadas: a) a aglutinação das equações do Erro Quadrático Médio de cada resposta através da sua soma ponderada; ou, b), a escolha da equação do Erro Quadrático Médio da resposta de maior importância como função objetivo, atribuindo às demais o caráter de restrições $[5,11]$.

Derringer e Suich [12] propuseram um conjunto de transformações para cada uma das $p$ respostas, resultando numa função individual denominada Desirability $\left(d_{i}\right), \operatorname{com} 0 \leq d_{i} \leq 1 . \mathrm{O}$ método permite incluir a importância individual de cada resposta $\left(w_{i}\right)$. Embora não tenha sido desenvolvido especificamente para problemas de Superfície de Resposta Dual, a formulação pode ser utilizada com esta conotação, em problemas do tipo NTB. Neste caso, a transformação Desirability pode ser escrita como o sistema de Equação 4 [12]:

$$
d_{i}\left[f_{i}(\mathbf{x})\right]=\left\{\begin{array}{ccc}
0 & \text { se } & f_{i}(\mathbf{x}) \leq f_{i}^{\min } \text { or } f_{i}(\mathbf{x})>f_{i}^{\max } \\
\frac{f_{i}(\mathbf{x})-f_{i}^{\min }}{T_{i}-f_{i}^{\min }} & \text { se } & f_{i}^{\min }<f_{i}(\mathbf{x}) \leq T \\
\frac{f_{i}^{\max }-f_{i}(\mathbf{x})}{f_{i}^{\max }-T_{i}} & \text { se } & T_{i}<f_{i}(\mathbf{x}) \leq f_{i}^{\max }
\end{array}\right.
$$

No sistema de equações (4), $f_{i}(x), f_{i}^{\min }, f_{i}^{\max }$ e $T_{i}$ são, respectivamente, o valor de $f_{i}(x)$ no ótimo, o limite inferior de 
especificação para a resposta, o limite superior de especificação e o alvo (valor nominal). Para estes problemas, as variáveis de resposta serão tratadas em faixas de aceitação. As transformações individuais das respostas podem ser combinadas utilizando-se uma média geométrica $(D)$, como demonstra a Equação 5:

$$
D=\left[\prod_{i=1}^{n} d_{i}{ }^{w_{i}}\left(\hat{Y}_{i}\right)\right]^{\frac{1}{W}}
$$

O método Desirability, entretanto, possui algumas limitações, destacando-se a dependência do método por uma escolha subjetiva das funções $d_{i}$ individuais e a negligência das variâncias das respostas e da estrutura de correlação entre elas $[1,13]$. No exemplo que será apresentado neste trabalho, embora as variáveis não sejam, em geral, do tipo NTB, esta suposição será estabelecida. Por exemplo, a penetração $(P)$ de um cordão de solda é tipicamente uma resposta do tipo Largeris-better (maximização). Entretanto, para fins desta aplicação, será tratada em termos de uma tolerância ou faixa.

\subsection{Erro Quadrático Médio Multivariado (EQMM)}

Baseado no Erro Quadrático Médio (EQM) empregado por Köksoy e Yalcinoz [5] e Lin e Tu [6], Paiva [7] e Paiva et al. $[18,19]$ propuseram uma adaptação capaz de considerar adequadamente a estrutura de correlação existente entre as respostas de interesse, a partir de combinações da Metodologia de Superfície de Resposta (MSR) e a Análise de Componentes Principais (ACP). Através desta combinação, gera-se uma superfície de resposta ajustada para os escores dos componentes principais, com a qual se compõe, então, o Erro Quadrático Médio Multivariado (EQMM).

A Análise de Componentes Principais (ACP) é uma técnica estatística multivariada capaz de explicar a estrutura de variânciacovariância existente em um conjunto de dados, utilizando-se combinações lineares não correlacionadas das variáveis originais, com o objetivo de se reduzir a dimensionalidade de vetores de entradas ou de saídas em determinados equacionamentos [14]. Esta técnica facilita sua interpretação, uma vez que, segundo Rencher [15], revela relacionamentos que não seriam previamente identificados com o conjunto original.

A idéia básica da ACP é que, embora $p$ componentes sejam necessários para se reproduzir a variabilidade total de um sistema de interesse, em geral, grande parte desta variabilidade pode ser representada por um pequeno grupo de $p$ componentes principais. Existe tanta informação em $p$ componentes principais quanto nas $p$ variáveis originais. Assim, o conjunto original de dados pode ser reduzido a poucos componentes principais.

Os componentes principais dependem somente da matriz de variância-covariância $\Sigma$ ou da matriz de correlação $\rho$ das variáveis $X_{1}, X_{2}, \ldots, X_{p}$. Seja o vetor aleatório $X^{T}=\left[X_{1}, X_{2}, \ldots, X_{p}\right]$, cuja matriz de variância-covariância $\Sigma$ possua autovalores $\lambda_{1} \geq \lambda_{2} \geq \ldots \geq \lambda_{p} \geq 0$. O primeiro componente principal $\left(P C_{l}\right)$ é a combinação linear que possuir a máxima variância [14]. Um conjunto de variáveis originais pode ser substituído por combinações lineares na forma de "escores" do componente principal.

O critério mais utilizado para se definir o número de componentes principais que devem ser utilizados é o critério de Kaiser [14], segundo o qual o autovalor do componente principal deve ser maior que um para representar o conjunto original. Além disso, a variância acumulada explicada deve ser superior a $80 \%$.

A otimização baseada no EQM é representada pela Equação 3. Entretanto, para problemas multivariados com um único componente principal, essa equação deve ser modificada conforme a Equação 6.

$$
\text { Minimizar } E Q M M_{P C}=\left(P C_{i}-\zeta_{P C_{i}}\right)+\lambda_{P C_{i}}
$$

$$
\text { sujeito } a: x^{T} x \leq \rho^{2}
$$

Onde $P C_{i}$ é a equação do $i$-ésimo componente principal e é $\zeta_{P C_{i}}$ o seu valor alvo, $\lambda_{P C_{i}}$ é o autovalor do $i$-ésimo componente principal e $x^{T} x \leq \rho^{2}$ é a restrição do espaço experimental para regiões esféricas (no caso de se utilizar um arranjo do tipo CCD para 3 fatores, $\rho=1,682$ [8]). Finalmente,

desejando-se a otimização de várias superfícies de resposta para componentes principais, tem-se:

$$
\begin{aligned}
& \text { Minimizar }\left[\prod_{i=1}^{n}\left(\operatorname{EQMM}_{P C_{i}} \mid \lambda_{i} \geq 1\right)\right]^{\frac{1}{n}} \\
& \text { sujeito } a: x^{T} x \leq \rho^{2}
\end{aligned}
$$

Onde $n$ é o número de funções EQMM consideradas de acordo com os componentes principais significativos.

Neste trabalho, o método EQMM será comparado ao tradicional método de Desirability, apenas para problemas do tipo alvo (NTB).

\section{Otimização do processo de soldagem FCAW}

Considerando que os processos de soldagem apresentam muitas características peculiares e específicas de processos multivariados, o método EQMM pode ser uma opção relevante na busca de condições de otimização. Neste trabalho, a abordagem proposta foi aplicada na otimização de um processo de soldagem FCAW (Flux Cored Arc Welding), cujo objetivo, originalmente investigado por Rodrigues [16], foi a análise da combinação ideal de parâmetros para a soldagem com arames tubulares rutílicos e seus resultados em algumas características específicas da solda. Aproveitaram-se os testes realizados onde foram feitas soldas de simples deposições em chapas de aço ABNT 1045 com dimensões de $75 \mathrm{~mm} \times 50 \mathrm{~mm} \times 9 \mathrm{~mm}$, 
Tabela 1. Matriz de experimentos (CCD) do processo de soldagem FCAW e pré-processamentos dos dados.

\begin{tabular}{|c|c|c|c|c|c|c|c|c|c|c|c|c|c|}
\hline \multicolumn{3}{|c|}{ Informações } & \multicolumn{3}{|c|}{ Fatores } & \multicolumn{5}{|c|}{ Respostas Originais } & \multicolumn{3}{|c|}{ Componentes Principais } \\
\hline Pontos & $n$ & $I(A)$ & $V$ & $V a$ & $d$ & $\begin{array}{c}P \\
(\mathrm{~mm}) \\
\end{array}$ & $\begin{array}{c}T D \\
(K g / h) \\
\end{array}$ & $R(\%)$ & $I C(\%)$ & $D(\%)$ & $P C 1$ & $P C 2$ & $P C 3$ \\
\hline \multirow{8}{*}{ 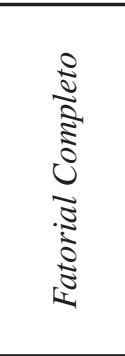 } & 1 & 235 & 29,0 & 10,0 & 15,0 & 1,89 & 3,62 & 82,49 & 32,10 & 38,76 & $-1,537$ & $-0,640$ & $-0,429$ \\
\hline & 2 & 246 & 36,0 & 10,0 & 15,0 & 1,93 & 3,49 & 79,47 & 21,68 & 44,26 & $-2,874$ & 0,499 & $-0,948$ \\
\hline & 3 & 286 & 29,0 & 14,0 & 15,0 & 2,40 & 5,14 & 86,83 & 55,49 & 30,26 & 2,321 & $-2,027$ & $-0,655$ \\
\hline & 4 & 300 & 36,0 & 14,0 & 15,0 & 2,88 & 5,28 & & & & 1,263 & 1,727 & $-0,136$ \\
\hline & 5 & 220 & 29,0 & 10,0 & 20,0 & 1,55 & 3,74 & 82,82 & 29,58 & 35,51 & $-1,684$ & $-1,224$ & 0,230 \\
\hline & 6 & 228 & 36,0 & 10,0 & 20,0 & 1,94 & 3,65 & 81,99 & 25,52 & 43,23 & $-1,932$ & 0,269 & $-0,370$ \\
\hline & 7 & 270 & 29,0 & 14,0 & 20,0 & 1,91 & 5,40 & 86,12 & 50,57 & 26,95 & 1,805 & $-2,655$ & $-0,019$ \\
\hline & 8 & 212 & 36,0 & 14,0 & 20,0 & 2,85 & 4,52 & 85,58 & 24,70 & 43,67 & 0,473 & 1,489 & $-0,174$ \\
\hline \multirow{6}{*}{ 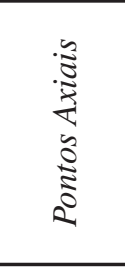 } & 9 & 255 & & & & 1,90 & & & & & & & \\
\hline & 10 & 276 & 38,4 & 12,0 & & 2,40 & & 84,10 & 23,80 & 42,11 & & 0,8 & $-0,066$ \\
\hline & 11 & 220 & 32,5 & 8,6 & 17,5 & 1,60 & 3,11 & 82,00 & 32,94 & 35,22 & $-2,237$ & $-1,476$ & $-0,270$ \\
\hline & 12 & 319 & 32,5 & 15,4 & 17,5 & 3,40 & 6,06 & 89,12 & 44,21 & 44,37 & 3,842 & 1,417 & $-0,652$ \\
\hline & 13 & 287 & 32,5 & 12,0 & 13,3 & 2,80 & 4,88 & 85,37 & 25,05 & 41,66 & 0,728 & 1,222 & $-0,150$ \\
\hline & 14 & 259 & 32,5 & 12,0 & 21,7 & 1,90 & 4,65 & 86,42 & 23,75 & 35,05 & 0,087 & $-0,426$ & 1,303 \\
\hline \multirow{6}{*}{ 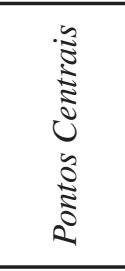 } & 15 & 262 & 32,5 & & & 1,90 & & & & 42,43 & & & \\
\hline & 16 & 267 & 32,5 & 12,0 & 17,5 & 2,28 & 4,25 & 83,51 & 24,91 & 42,19 & $-0,746$ & 0,636 & $-0,192$ \\
\hline & 17 & 262 & 32,5 & 12,0 & 17,5 & 2,30 & 4,37 & 84,82 & 26,85 & 42,65 & $-0,236$ & 0,657 & 0,078 \\
\hline & 18 & 259 & 32,5 & 12,0 & 17,5 & 2,15 & 4,18 & 86,49 & 26,79 & 39,80 & $-0,048$ & 0,182 & 0,791 \\
\hline & 19 & 260 & 32,5 & 12,0 & 17,5 & 2,30 & 4,22 & 85,79 & 25,06 & 44,30 & $-0,217$ & 0,947 & 0,428 \\
\hline & 20 & 278 & 32,5 & 12,0 & 17,5 & 2,35 & 4,50 & 86,08 & 27,15 & 45,10 & 0,193 & 1,029 & 0,351 \\
\hline \multirow{4}{*}{\multicolumn{3}{|c|}{$\begin{array}{l}\text { Processamento dos dados } \\
\text { para aplicação do EQMM }\end{array}$}} & \multirow{3}{*}{\multicolumn{3}{|c|}{$\begin{array}{c}\text { Média da coluna: } \\
\text { Desvio Padrão da coluna: } \\
\text { Alvo }\left(\zeta_{Y}\right):\end{array}$}} & 2,232 & 4,442 & 84,865 & 31,176 & 39,477 & 0,000 & 0,000 & 0,000 \\
\hline & & & & & & 0,468 & 0,714 & 2,225 & 10,211 & 5,884 & 1,623 & 1,369 & 0,548 \\
\hline & & & & & & 2,700 & 4,650 & 85,000 & 26,000 & 40,000 & $-0,447$ & $-0,829$ & 0,251 \\
\hline & & & \multicolumn{3}{|c|}{$Z\left(Y_{i} \mid \zeta_{X}\right):$} & 1,000 & 0,292 & 0,061 & $-0,507$ & 0,089 & $-0,276$ & $-0,606$ & 0,458 \\
\hline
\end{tabular}

Nota: Valores em negrito representam os alvos calculados para os escores de componentes principais.

polaridade reversa $(\mathrm{CC}+)$, utilizando arame AWS E71T-1, diâmetro $1,2 \mathrm{~mm}$, velocidade de soldagem de $50 \mathrm{~cm} / \mathrm{min}$ e proteção gasosa de $\mathrm{CO}_{2}$, com vazão de $15 \mathrm{l} / \mathrm{min}$ e inclinação da tocha de $70^{\circ}$ no sentido puxando, procurando-se assim aumentar a penetração da solda. Utilizou-se uma fonte de soldagem multiprocessos Inversal 300, com comando digital e funcionamento no modo normal. A tocha de soldagem foi acoplada a um carro com ajuste de velocidade e a um sistema mecânico que permitiu o ajuste de posicionamento nas condições especificadas. Para avaliar o comprimento do arame consumido na realização de cada cordão, foi utilizado um tacômetro, acoplado à fonte de soldagem que permitia também avaliar o tempo de arco aberto. Utilizou-se em todos os testes, um software para aquisição dos dados de tensão e corrente, tornando-se possível observar as características dinâmicas de transferência das gotas.

Considerando-se como fatores a Tensão $(V)$, a Velocidade de Alimentação do Arame ( $V a$ ) e a Distância do Bico de Contato da Peça $(d)$, registrando-se as respostas de Penetração $(P)$, Taxa de Deposição (TD), Rendimento ${ }^{\circledR}$, Índice de Convexidade (IC) e Diluição $(D)$.
A taxa de deposição (TD, $\mathrm{kg} / \mathrm{h}$ ) foi determinada pela equação (10):

$$
\mathcal{D}=\frac{3,6 \times\left(m_{f}-m_{i}\right)}{t}
$$

onde: $T D$ - taxa de deposição em $(\mathrm{kg} / \mathrm{h}) ; m_{f}$ - massa do corpo de prova após a soldagem $(\mathrm{g}) ; m_{i}$ - massa do corpo de prova antes a soldagem (g) e $t$ o tempo de soldagem (s).

O rendimento ${ }^{\circledR}$ foi determinado pela relação percentual entre a taxa de deposição e a taxa de fusão sendo determinado pela equação (11):

$$
R=\frac{\left(m_{f}-m_{i}\right)}{d_{L} \times L} \times 100
$$

onde: $d_{L}$ é a densidade linear do arame utilizado $(\mathrm{g} / \mathrm{m})$ e $L$ o comprimento do arame fundido (m).

Após executadas as soldagens, os corpos de prova foram seccionados em dois locais distintos para diminuir os erros de medição. Posteriormente, os mesmos foram polidos e atacados quimicamente com Nital $4 \%$ e avaliados através de 
um analisador de imagem Olympus - Analysis Digital Imaging Solutions, registrando-se os valores de $P, h, b, S_{p}, S_{t} . I C$ e $D$ foram calculados, respectivamente, através das equações (12) e (13).

$$
I C=\frac{h}{b} \times 100
$$

onde: $\quad h$ - reforço do cordão, mm, e $b$ - largura do cordão, $\mathrm{mm}$.

$$
D=\frac{S_{p}}{S_{t}} \times 100
$$

onde: $S_{p}$ - área correspondente a penetração do cordão, $\mathrm{mm}^{2}$, $S_{t}$ - área correspondente ao cordão total, $\mathrm{mm}^{2}$.

Utilizando-se um arranjo de superfície de resposta do tipo CCD para 3 fatores ( $V, V a$ e $d$ ) como base para a realização dos experimentos, foram obtidos os resultados da Tabela 1.

$\mathrm{Na}$ Tabela 1 constam também os valores de corrente medidos durante o processo de soldagem. Vale ressaltar que, em função da fonte ser do tipo tensão constante, ao se regular os parâmetros de tensão e velocidade de alimentação do arame, a corrente de soldagem se auto-ajusta para garantir o comprimento do arco e consequentemente o valor de tensão.

Para se avaliar se os dados do processo FCAW são adequados à aplicação do método EQMM, procedeu-se uma análise de correlação entre as respostas obtidas, tal como descrito pelo fluxograma da Figura 1. Através da análise de correlação (Tabela 2), constatou-se haver forte correlação da resposta $P$ em relação a $T D$ e $R$, correlação moderada entre $P$ e $D$, e correlação inexistente entre $P$ e $I C$; forte correlação da resposta $T D$ em relação a $R$, forte correlação da resposta $I C$ em relação a $D$, e correlação moderada de $T D$ em relação a $I C$. Para as demais respostas, as correlações são insignificantes ou não existem. Neste caso, não foram analisadas correlações entre as variáveis de entrada (fatores) porque arranjos experimentais como o CCD (Tabela 1), arranjos fatoriais ou Taguchi são arranjos ortogonais. Isto significa que a correlação entre variáveis de entrada é nula [8].

Tabela 2. EQMM: correlação entre as respostas do processo FCAW.

\begin{tabular}{c|cccc}
\hline & $P$ & $T D$ & $R$ & $I C$ \\
\hline \multirow{3}{*}{$T D$} & 0,721 & & & \\
& 0,000 & & & \\
\multirow{3}{*}{$R$} & 0,541 & 0,852 & & \\
& 0,014 & 0,000 & & \\
$I C$ & $-0,027$ & 0,447 & 0,416 & \\
& 0,909 & 0,048 & 0,068 & \\
$D$ & 0,491 & $-0,069$ & $-0,104$ & $-0,754$ \\
& 0,028 & 0,773 & 0,662 & 0,000 \\
\hline
\end{tabular}

A estrutura de correlação verificada entre as variáveis citadas viabiliza a aplicação do método EQMM. Dada a existência de correlação entre as respostas, procedeu-se à análise de componentes principais, tal como descrito pela Tabela 3. Desta análise, conclui-se que três componentes principais conseguem explicar mais de $96 \%$ da variância acumulada. Portanto, o método EQMM pode ser implementado utilizando-se apenas 3 componentes.

\begin{tabular}{|c|c|c|c|c|c|c|c|}
\hline & Autovalores & $\lambda_{i}$ & 2,5928 & 1,9075 & 0,3049 & 0,1301 & 0,0646 \\
\hline & Proporção & $\%$ & 0,519 & 0,381 & 0,061 & 0,026 & 0,013 \\
\hline & Acumulado & $\%$ acum. & 0,519 & 0,900 & 0,961 & 0,987 & 1,000 \\
\hline \multicolumn{8}{|c|}{ Autovetores da Matriz de Correlação } \\
\hline Variáveis & Alvos & $Z(A l v o s)$ & $P C_{1}$ & $P C_{2}$ & $\mathrm{PC}_{3}$ & $\mathrm{PC}_{4}$ & $P C_{5}$ \\
\hline$P$ & 2,70 & 1,0001 & $-0,427$ & $-0,469$ & 0,529 & $-0,145$ & $-0,545$ \\
\hline$T D$ & 4,65 & 0,2922 & $-0,600$ & $-0,078$ & 0,008 & $-0,441$ & 0,663 \\
\hline$R$ & 85,00 & 0,0609 & $-0,566$ & $-0,026$ & $-0,701$ & 0,314 & $-0,298$ \\
\hline$I C$ & 26,00 & $-0,5069$ & $-0,356$ & 0,540 & 0,476 & 0,583 & 0,122 \\
\hline \multirow[t]{2}{*}{$D$} & 40,00 & 0,0890 & 0,100 & $-0,694$ & 0,039 & 0,589 & 0,400 \\
\hline & & $\operatorname{Alvos}\left(\zeta_{Y}\right)$ & $-0,4475$ & $-0,8289$ & 0,2509 & $-0,176$ & 0,043 \\
\hline
\end{tabular}

Tabela 3. Análise de Componentes Principais do processo FCAW e cálculo de alvos para os componentes. 
Os valores definidos para os alvos dos componentes principais $\left(\zeta_{Y}\right)$ bem como os valores para os escores de componentes

(colunas $P C_{1}, P C_{2}$ e $P C_{3}$ da Tabela 1) foram calculados multiplicando-se os autovetores de cada componente principal pelo valor padronizado $(Z)$ de cada resposta de interesse, tal como recomendam Johnson e Wichern [14]. Tanto para a ACP quanto para a geração e análise do arranjo experimental de superfície de resposta, foi utilizado o software estatístico Minitab® 15.0. Os escores de componentes principais e os níveis das variáveis mostrados na Tabela 1 são calculados diretamente pelo software.

Uma vez computadas as respostas obtidas experimentalmente com o arranjo CCD, procedeu-se à análise estatística de significância dos modelos quadráticos completos e a determinação dos coeficientes dos modelos, tal como sugere o fluxograma da Figura 1. Esta análise foi feita tanto para as respostas originais quanto para cada um dos componentes principais. Considerandose o nível de significância $\alpha=5 \%$, todos os modelos quadráticos

completos apresentados na Tabela 4 demonstraram-se estatisticamente significativos, embora os ajustes obtidos não sejam, em geral, muito altos. Destaca-se, porém, que o ajuste realizado para o primeiro componente principal obteve $R^{2}$ ajustado de quase $88 \%$, mostrando que a análise feita utilizandose os escores deste componente explica grande parte da variação que foi observada nas cinco respostas originais. Tanto para as respostas originais quanto para os componentes principais, alguns termos não apresentaram significância individual; entretanto, sua remoção dos modelos não melhorou o ajuste ou reduziu a variabilidade. Portanto, de acordo com o princípio da Hierarquia [8], mantiveram-se os modelos quadráticos completos.

\subsection{Análise de resultados}

Para aplicar os métodos EQMM e Desirability, foram estabelecidos os limites de especificação e os alvos mostrados na Tabela 5, considerando para isto condições que se julgavam mais adequadas para um cordão otimizado, bem como informações disponíveis na literatura [17]. Vale ressaltar que o rendimento máximo foi fixado em $90 \%$ devido ao arame ser tubular e, desta forma, haver formações de escória e respingos durante a soldagem, o que compromete o rendimento. Os alvos foram estabelecidos como metade da tolerância.

Tabela 4. FCAW: modelos quadráticos completos para as respostas.

\begin{tabular}{ccccccccc}
\hline Termo & $P$ & $T D$ & $R$ & $I C$ & $D$ & $P C_{1}$ & $P C_{2}$ & $P C_{3}$ \\
\hline$b_{0}$ & 2,219 & 4,355 & 85,509 & 26,354 & 42,705 & $-0,167$ & 0,620 & 0,397 \\
$V$ & 0,197 & $-0,075$ & $-0,640$ & $-8,333$ & 5,157 & $-0,465$ & 1,215 & $-0,065$ \\
$V a$ & 0,422 & 0,791 & 2,179 & 4,901 & $-0,026$ & 1,765 & 0,329 & $-0,008$ \\
$d$ & $-0,173$ & $-0,044$ & 0,253 & $-0,526$ & $-1,477$ & $-0,116$ & $-0,326$ & 0,313 \\
$V^{2}$ & $-0,059$ & 0,027 & $-0,450$ & 3,629 & $-2,656$ & 0,047 & $-0,567$ & $-0,214$ \\
$V a^{2}$ & 0,065 & 0,018 & $-0,306$ & 4,222 & $-0,782$ & 0,169 & $-0,246$ & $-0,368$ \\
$d^{2}$ & 0,012 & 0,082 & $-0,188$ & $-0,790$ & $-1,291$ & 0,029 & $-0,095$ & $-0,001$ \\
$V x V a$ & 0,124 & $-0,065$ & 0,314 & $-5,098$ & 2,296 & $-0,101$ & 0,658 & 0,185 \\
$V x d$ & 0,101 & $-0,123$ & 0,306 & 1,235 & 0,509 & 0,102 & 0,093 & $-0,094$ \\
$V a x d$ & $-0,024$ & $-0,098$ & $-0,501$ & $-0,955$ & $-0,061$ & $-0,263$ & $-0,007$ & $-0,080$ \\
$R^{2} a d j$. & $78,6 \%$ & $86,3 \%$ & $70,0 \%$ & $96,8 \%$ & $70,0 \%$ & $87,7 \%$ & $79,7 \%$ & $51,4 \%$ \\
\hline
\end{tabular}

Tabela 5. Limites de especificação dos parâmetros do processo FCAW

\begin{tabular}{cccc}
\hline Respostas & Mínimo & Alvo & Máximo \\
\hline Penetração (mm) & 1,9 & 2,7 & 3,5 \\
Taxa de deposição (kg/h) & 3,8 & 4,6 & 5,5 \\
Rendimento (\%) & 80 & 85 & 90 \\
Índice de convexidade (\%) & 22 & 26 & 30 \\
Diluição (\%) & 35 & 40 & 45 \\
\hline
\end{tabular}


Utilizando-se as equações de 3 componentes, o EQMM pode ser formulado como um problema de otimização não-linear, tal que:

$$
\begin{array}{cl}
\text { Minimizar } & \left.E Q M M_{T}=\left\{\left[\left(P C_{1}-\zeta_{P C_{1}}\right)^{2}+\lambda_{1}\right] k\left(P C_{2}-\zeta_{P C_{2}}\right)^{2}+\lambda_{2}\right] k\left[\left(P C_{3}-\zeta_{P C_{3}}\right)^{2}+\lambda_{3}\right]\right\}^{1 / 3} \\
\text { Sujeito a: } & \mathbf{x}^{T} \mathbf{x} \leq \rho^{2}=V^{2}+V a^{2}+d^{2} \leq(1,682)^{2} \\
\text { Com: } & \zeta_{P C_{i}}=e_{1 i}\left[Z\left(P \mid \zeta_{P}\right)\right]+e_{2 i}\left[Z\left(T D \mid \zeta_{T D}\right)\right]+e_{3 i}\left[Z\left(R \mid \zeta_{R}\right)\right]+e_{4 i}\left[Z\left(I C \mid \zeta_{I C}\right)\right]+e_{5 i}\left[Z\left(D \mid \zeta_{D}\right)\right. \\
& i=1,2, \ldots, p .
\end{array}
$$

Onde: $Z$ representa o valor padronizado da $i$-ésima resposta considerando o valor alvo $\zeta_{Y}$, tal que $Z\left(Y_{i} \mid \zeta_{H}\right)=\left[\left(\zeta_{Y}\right)-\mu_{\Psi}\right] .\left(\sigma_{I}\right)^{-1}$. Os valores numéricos dos alvos padronizados $Z\left(\left.Y_{i}\right|_{\zeta_{Y}}\right)$ foram citados na última linha da Tabela 3. Os resultados obtidos estão demonstrados na Tabela 7. Este sistema de otimização não-linear pode ser resolvido por vários algoritmos e softwares. Neste trabalho, empregou-se o algoritmo GRG (Gradiente Reduzido Generalizado) disponível no MS-Excel Solver. Os resultados do método Desirability foram obtidos com o pacote Response Optimizer do Minitab® 15.0, embora também sejam facilmente obtidos com o Solver.

Tabela 6. Comparação entre os métodos EQMM e Desirability.

\begin{tabular}{lcccccccc}
\hline & $V$ & $V a$ & $d$ & $P$ & $T D$ & $R$ & $I C$ & $D$ \\
\cline { 2 - 8 } & $\mathrm{V}$ & $\mathrm{m} / \mathrm{min}$ & $\mathrm{mm}$ & $\mathrm{mm}$ & $\mathrm{kg} / \mathrm{h}$ & $\%$ & $\%$ & $\%$ \\
\hline Mínimo & 26,6 & 8,6 & 13,3 & 1,90 & 3,80 & 80,00 & 22,00 & 35,00 \\
Máximo & 38,4 & 15,4 & 21,7 & 3,50 & 5,50 & 90,00 & 30,00 & 45,00 \\
Desirability & $\mathbf{3 7 , 9}$ & $\mathbf{1 4 , 3}$ & $\mathbf{1 3 , 3}$ & 3,53 & 5,79 & 86,24 & 21,61 & 49,77 \\
EQMM & $\mathbf{3 2 , 2}$ & $\mathbf{1 2 , 4}$ & $\mathbf{1 3 , 6}$ & 2,62 & 4,76 & 84,96 & 27,67 & 41,45 \\
\hline
\end{tabular}

Nota-se que a solução apresentada pelo EQMM (em negrito) atinge, para todas as respostas, valores dentro dos limites de especificação. O Desirability, por sua vez, ultrapassa os limites máximos para as respostas $P$ (penetração), $T D$ (taxa de deposição) e $D$ (diluição), e o limite mínimo para a resposta $I C$ (índice de convexidade). Esses resultados apontam para uma sensível melhoria na solução, quando o EQMM é empregado, demonstrando a sua eficiência em otimização simultânea e ratificando a boa adequação da proposta à otimização simultânea de múltiplas características. Um gráfico de contorno foi gerado para os resultados apurados pelo EQMM, a fim de se verificar a localização do valor ótimo em relação às respostas obtidas pelo modelo, comparativamente aos resultados obtidos pelo Desirability, conforme demonstra a Figura 2.

\subsection{Experimentos de Confirmação}

Para avaliar a qualidade dos resultados obtidos pelos dois métodos, realizaram-se experimentos de confirmação com cada uma das duas soluções encontradas. Os resultados foram resumidos na Tabela 7 a seguir.

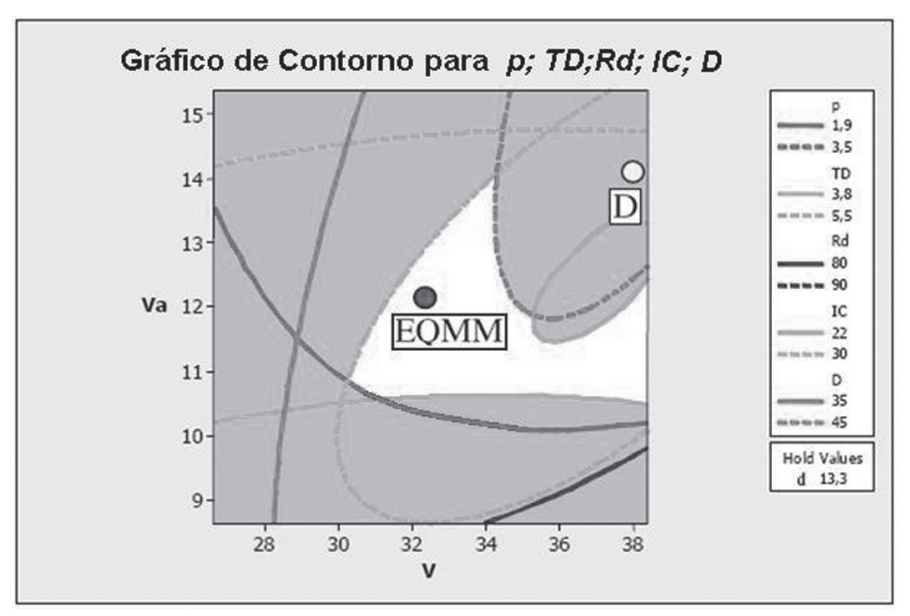

Figura 2. Gráfico de contorno para EQMM do processo de soldagem FCAW Legenda: (D) Desirability; (EQMM) método multivariado. 
Tabela 7 - Análise comparativa dos experimentos de confirmação.

\begin{tabular}{|c|c|c|c|c|c|c|}
\hline Método & & $P$ & $T D$ & $R$ & $I C$ & $D$ \\
\hline & Alvos & 2,70 & 4,65 & 85,00 & 26,00 & 40,00 \\
\hline \multirow{4}{*}{ 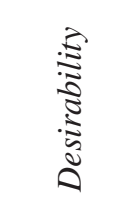 } & Real & 2,65 & 4,95 & 83,71 & 24,4 & 45,05 \\
\hline & Teórico & 3,53 & 5,79 & 86,25 & 21,61 & 49,77 \\
\hline & Erro (Real x Teórico) & $-24,90 \%$ & $-14,50 \%$ & $-2,94 \%$ & $12,90 \%$ & $-9,48 \%$ \\
\hline & Erro (Real x Alvo) & $-0,05 \%$ & $0,30 \%$ & $-1,29 \%$ & $-1,60 \%$ & $5,05 \%$ \\
\hline \multirow{4}{*}{ 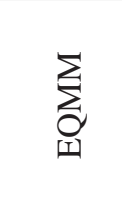 } & Real & 2,5 & 4,5 & 84,01 & 28,1 & 40,5 \\
\hline & Teórico & 2,62 & 4,76 & 84,96 & 27,67 & 41,45 \\
\hline & Erro (Real x Teórico) & $-4,60 \%$ & $-4,50 \%$ & $-1,12 \%$ & $1,60 \%$ & $-2,20 \%$ \\
\hline & Erro (Real x Alvo) & $-0,20 \%$ & $-0,15 \%$ & $-0,99 \%$ & $2,10 \%$ & $0,50 \%$ \\
\hline
\end{tabular}

Da Tabela 7, depreende-se que o método EQMM proporcionou valores reais e teóricos mais próximos e, em geral, com menores distâncias em relação ao valor do alvo, demonstrando sua eficácia em problemas do tipo Nominal, com variáveis de resposta correlacionadas.

Os experimentos de confirmação permitiram avaliar comparativamente os métodos EQMM e Desirability, expressando os erros percentuais observados entre os valores teóricos obtidos pela aplicação de cada um dos modelos e seus valores reais, e dos valores reais em relação a seus alvos. Os baixos erros percentuais evidenciados denotam a fiabilidade do modelo EQMM, na busca por parâmetros ideais de processos.

\section{Conclusão}

Este trabalho apresentou o método de otimização de múltiplas respostas correlacionadas denominado EQMM. Este método pode ser empregado em todos os processos que possuírem características do tipo NTB, cujas características de saída apresentem forte ou moderada estrutura de correlação. Como pôde ser observado em relação ao processo FCAW, o EQMM permitiu o atendimento dos limites especificados para todas as respostas, sem ultrapassar a restrição do espaço experimental imposta, enquanto considerou adequadamente a estrutura de correlação, apresentando erros percentuais em relação aos valores de confirmação e em relação ao alvo satisfatoriamente pequenos, em detrimento dos resultados do método Desirability. Embora os resultados obtidos neste estudo não possam ser extrapolados ou generalizados, sua aplicação em problemas de otimização de processos de manufatura com múltiplas características pode ser recomendada.

\section{Agradecimentos}

Os autores gostariam de agradecer o apoio da Capes, $\mathrm{CNPq}$ e FAPEMIG, na realização deste trabalho.

\section{Referências Bibliográficas}

[1] Wu, F. C., 2005, "Optimization of correlated multiple quality characteristics using desirability function", Quality Engineering, v 17, n 1, pp. 119-126.

[2] Khuri, A. I.; Cornell, J. A., 1996, "Response surfaces: designs and analyse", Marcel Dekker Inc, 2 ed, New York, USA, 510p.

[3] Khuri, A. I.; Conlon, M., 1981, "Simultaneous optimization of multiple responses represented by polynomial regression functions", Technometrics, v 23, n 4, pp. 363-375.

[4] Bratchell, N., 1989, "Multivariate response surface modelling by principal components analysis", Journal of Chemometrics, $\mathrm{v}$ 3, pp. 579-588.

[5] Köksoy, O.; Yalcinoz, T., 2006, "Mean square error criteria to multiresponse process optimization by a new genetic algorithm", Appl. Math. Comput., n. 175, p. 1657-1674.

[6] Lin, D. K. J.; Tu, W., 1995, "Dual response surface optimization”, Journal of Quality Technology 27:34-39.

[7] Paiva, E. J., 2008, "Otimização de processo de manufatura de múltiplas respostas baseada em índices de capacidade", Dissertação de Mestrado, Universidade Federal de Itajubá, Instituto de Engenharia de Produção, Itajubá, MG.

[8] Montgomery, D. C., 2001, "Design and analysis of experiments", Fourth ed., Wiley, New York.

[9] Montgomery, D. C.; Runger, G. C., 2003, "Estatística aplicada e probabilidade para engenheiros", LTC - Livros Técnicos e Científicos Editora S.A., 2 ed., p. 570.

[10] Vining, G. G.; Myers, R. H., 1990, "Combining Taguchi and response surface philosophies: a dual response approach", Journal of Quality Technology 22:38-45.

[11] Köksoy, O., 2007, "A nonlinear programming solution to robust multiresponse quality problem", Appl. Math. Comput., v. 6, n. 23.

[12] Derringer, G.; Suich, R., 1980, "Simultaneous optimization of several response variables", Journal of Quality Technology, v 12, n 4, pp.214-219.

[13] Ko, Y. H.; Kim, K. J., Jun, C. H., 2005, "A new loss function-based method for multiresponse optimization", Journal of Quality Technology, v 37, n 1, pp. 50-59.

[14] Johnson, R. A.; Wichern, D. W., 1981, "Applied multivariate statistical analysis", New Jersey: Prentice-Hall Inc., 5 ed., $797 \mathrm{p}$.

[15] Rencher, A.C., 2002, "Methods of multivariate analysis", John Wiley and Sons, 2 ed., 740p.

[16] Rodrigues, L. O., 2006, “Análise e otimização de parâmetros 
na soldagem com arame tubular", Dissertação de Mestrado, Universidade Federal de Itajubá, Instituto de Engenharia Mecânica, Itajubá, MG, 80 p.

[17] Silva, C.R.; Ferraresi, V.A.; Scotti, A., 2000, "A quality and cost approach for welding process selection", Journal of the Braz. Soc. Mechanical Sciences, vol. xxii, no. 3, pp. 389-398.

[18] Paiva, A.P.; Paiva, E.J.; Ferreira, J.R.;Balestrassi, P.P.;Costa, S.C., 2009, "A multivariate mean square erro optimization of AISI 52100 hardened steel turning", International Journal of Advanced Manufacturing Technology, DOI 10.1007/s00170008-1745-5.

[19] Paiva, A.P.; Costa, S.C Paiva, E.J.; Balestrassi, P.P.; Ferreira, J.R.; 2010, "Multi-objective optimization of pulsed gas metal arc welding process based on weighted principal component scores", International Journal of Advanced Manufacturing Technology, DOI 10. 10.1007/s00170-009-2504-y. 